\title{
Evidence of a magnetic sheath around a jet from NGC 6543
}

\author{
Aden B. Meinel and Barbara Meinel \\ 2548 Eclipsing Stars Drive Henderson, NV 89044, USA \\ email: ammeinel@cox.net; ameinel@optics.arizona.edu
}

\begin{abstract}
We present observational evidence that NGC 6543 produced a jet of cosmic rays that irradiated the Earth recorded as cosmogenic10Be found ice cores. This identification shows that the jet was accompanied by a magnetic field of sufficient strength to travel $220 \mathrm{pc}$ and retain evidence of the celestial coordinates of the source object.
\end{abstract}

Keywords. Stars: AGB and post-AGB - accretion - planetary nebulae

The basic data of the flux of cosmogenic isotope 10Be is from the Greenland GRIP data archive and is corrected for the dilution effect of variable annual ice accumulation. Our attention was drawn to a set of sinusoidal oscillations indicated by the five black dots.

Fig. 1(A). We recognized that the change of angle of the Earth with regard to the sky as the result of precession would produce no signature of precession on an the isotropic flux of Galactic cosmic rays. The 21 ky period of lunar-solar precession therefore indicated the presence of a significant flux of cosmic rays from a point source.

Fig. 1(B). We calculated the effect of the combined $21 \mathrm{ky}$ lunar-solar plus $120 \mathrm{ky}$ apsidal precession as a function of Right Ascension of the point source. Fig. 1(B) shows the significant changes in the attenuation.

Fig. 1(C). The data for 10Be has been corrected in Fig. 1(C) for changing attenuation as the geomagnetic field. Note that the prominent surge at $40 \mathrm{kyBP}$ in Fig. 1(A) has merged imperceptibly into the general pattern of wide variations of the flux of $10 \mathrm{Be}$, but that the several oscillations are unchanged. The best fit of the calculated attenuation patterns is for a source close to $18 \mathrm{~h}$ Right Ascension and +65 Declination This is very close to the position of NGC 6543. Our finding is that this planetary nebula was the source of the cosmic rays that irradiated the Earth. Our calculation of the absolute fractional variation of 10Be best-fitted in Fig. 1(C) also defines the invariant flux of Galactic cosmic rays of $0.510 \mathrm{Be}$ particles/square centimeter per year.

The data pertaining to NGC 6543 of importance in our paper are:

A parallax of $0.045 \pm 0.021$ arcseconds was determined from three years of trigonometric observations by Gatewood and his associates at Allegheny Observatory (2008), thus a distance of 220 parsecs. The radius of curvature of a typical cosmic ray particle in the Galactic magnetic field however is less than 100th parsec. Thus no cosmic rays could reach the solar vicinity from NGC 6543 unless the cosmic rays were protected by a magnetic field accompanying the jet of cosmic rays.

The HST image in Fig. 1(C)a shows the central active region of NGC 6543 in negative format to show better the pairs of emission bubbles ejected in opposite directions as shown in Fig. 1(C)b. The schematic diagram (top) shows the orbital geometry consistent with our identification of NGC 6543 as the source of continual jetting of cosmic rays.

We find that the central object of NGC 6543 must consist of an Aging Giant Branch (AGB) star (S1) emitting plasma feeding a compact companion (S2), a neutron or white 


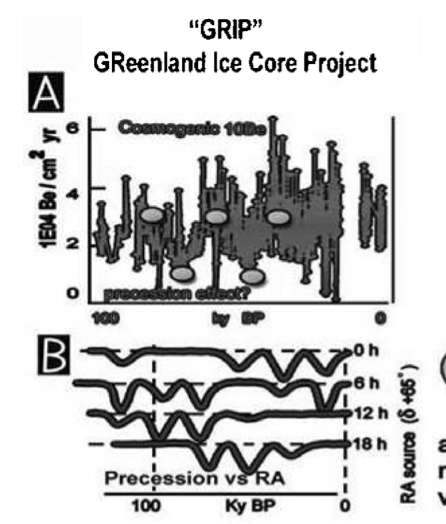

\section{NGC 6543: AGB S1 feeds S2 accretion disc \\ Evidence of a magnetic sheath around a jet from NGC 6543 Aden B. Meinel, Marjorie P. Meinel \& Barbara Meinel}

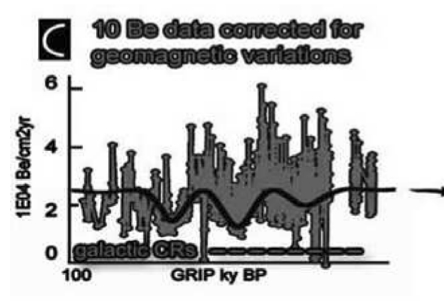

Best fit at $18.2 \mathrm{~h}+65^{\circ}$
$=\mathrm{NGC} 6543$ at $18 \mathrm{~h}+65^{\circ}$
trig $\pi=0.045 \pm 0.021 \mathrm{asec}$
(Gatewood et al)
distance $=220 \mathrm{pC}$
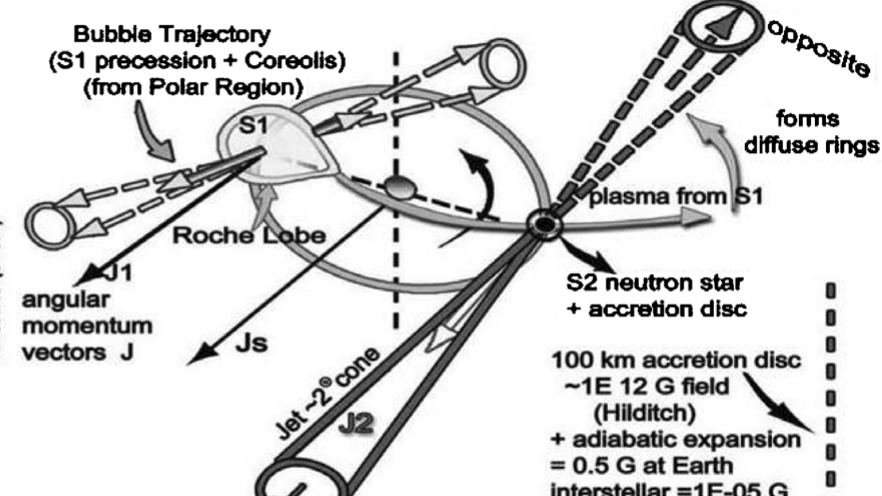

(Hilditch)

+ adiabatic expansion
$=0.5 \mathrm{G}$ at Earth

interstellar $=1 \mathrm{E}-05 \mathrm{G}$

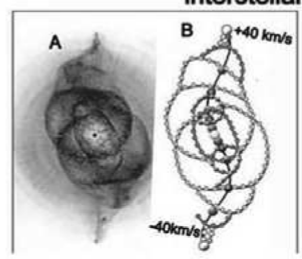

Jetting lasted

$\sim 150,000$ yrs

Figure 1. NGC 6543. AGB star S1 feeds S2 accretion disk.

dwarf star, plus an accretion disc. Part of this stream of plasma is acquired by the accretion disc, the balance escaping (Boyarchuk et al.) to be spread by orbital rotation into the diffuse rings shown in the HST image (Fig. 1(C)a).

The rotation axes of both stars and the system are parallel and indicated by their invariant angular momentum vectors $\mathrm{J} 1$, Js, and $\mathrm{J} 2$. The jet is aimed throughout its orbit toward the observer. The outflow of pairs of bubbles is from the polar regions of $\mathrm{S} 1$. The direction of outflow is affected by both precession of S1 and the Coriolis force. The direction of successive pairs of bubbles relative to the observer are unaffected by motion of S1 around its binary orbit, thus forming the vertical chain of bubbles shown in Fig. 1(C)b.

The survival of the jet in traversing the interstellar magnetic field depends on the very strong magnetic field of either a white dwarf or neutron star of $10^{12}$ Gauss. If the divergence angle of the jet is $2^{\circ}$, adiabatic expansion of the magnetic energy in the jet after a travel of 220 parsecs will still be 0.5 Gauss, much stronger than the $10^{-5}$ Gauss of the interstellar field.

Thus our conclusion is that a jet of cosmic rays from NGC 6543 can survive to reach the Earth and still indicate from whence they came. The astrophysical surprise is that the jetting of NGC 6543 lasted longer than 150,000 years. 